\title{
RECIPROCAL INHIBITION IN THE MOTOR NERVOUS SYSTEM OF THE NEMATODE ASCARIS: DIRECT CONTROL. OF VENTRAL INHIBITORY MOTONEURONS BY DORSAL EXCITATORY MOTONEURONS ${ }^{1}$
}

\author{
J. P. WALROND ${ }^{2}$ AND A. O. W. STRETTON ${ }^{3}$ \\ Department of Zoology, University of Wisconsin, Madison, Wisconsin 53706 \\ Received May 28, 1981; Revised July 23, 1984; Accepted July 25, 1984
}

\begin{abstract}
In previous physiological experiments (Stretton, A. O. W., R. M. Fishpool, E. Southgate, J. E. Donmoyer, J. P. Walrond, J. E. R. Moses, and I. S. Kass (1978) Proc. Natl. Acad. Sci. U. S. A. 75: 3493-3497), we have shown that the dorsal cord of the nematode Ascaris lumbricoides includes the processes of three types of dorsal excitatory (DE) motoneurons and one type of ventral inhibitory (VI) motoneuron. Ultrastructural studies have revealed that the axons of the DE motoneurons make monosynaptic contacts with the dorsal processes of VI motoneurons. In this paper, we describe a physiological preparation with which to investigate the properties of these synapses. We show that activation of a DE neuron can excite a VI neuron producing inhibition in ventral muscle cells shortly after dorsal muscle cells are excited, thus mediating reciprocity between dorsal and ventral muscles. Each VI dendrite receives input from four or five DE neurons; activation of any one of these DE neurons is sufficient to activate the VI neuron.
\end{abstract}

The somatic musculature of Ascaris lumbricoides consists of dorsal and ventral sheets of muscle cells which are separated by the lateral lines. Each muscle sheet is separately innervated by processes of motoneurons in the dorsal and ventral nerve cords, respectively. We have previously shown that the nerve cords contain seven different, anatomically defined classes of motoneurons (Stretton et al., 1978). The cell bodies of motoneurons are distributed along the length of the ventral nerve cord in a repeating pattern. Each repeating unit or segment contains a basic set of 11 neurons and includes one copy of neuron types DE2, DE3, and DI, and two copies of neuron types DE1, VI, V-1, and V-2 (Fig. 2 in Walrond et al., 1985). All motoneurons have a process in the ventral nerve cord. Five of the seven classes of motoneurons send individual neuron processes around the body wall and into the dorsal cord. These ventrodorsal processes are called commissures (a term which is of long-standing usage in the nematode literature (Hesse, 1892; Bullock and Horridge, 1965)). In the previous paper (Walrond et al., 1985) we used a dissected preparation in which dorsal

\footnotetext{
${ }^{1}$ We thank A. Chambers, A. Aaroen, C. Hughes, and D. Chandler for their help in preparing the manuscript. We also thank Dr. C. D. Johnson for the benefit of many stimulating discussions, and both Dr. Johnson and Dr. C. Kung for critical reading of the manuscript. This work was supported by United States Public Health Service Grants NS 10509 and AI 15429, National Science Foundation Grant BNS 7609641, a Sloan Fellowship to A. O. W. S., and the Research Fund of the Graduate School, University of Wisconsin, Madison.

${ }^{2}$ Present address: Laboratory of Neurobiology, National Institute of Neurological and Communicative Disorders and Stroke, Marine Biological Laboratory, Woods Hole, MA 02543.

${ }^{3}$ To whom correspondence should be addressed.
}

and ventral nerve cords remain linked by only a single commissure to investigate the physiological sign of commissural motoneurons. We showed that neuron types DE1, DE2, and DE3 are dorsal excitatory motoneurons, while the DI and VI neurons are dorsal and ventral inhibitors, respectively.

The region of synaptic interaction between a motoneuron and the muscle cells it innervates defines the output region of the motoneurons. The output regions of the dorsal excitatory motoneurons, DE1, DE2, and DE3, lie in the dorsal cord; DE dendrites lie in the ventral cord where they receive anatomically defined synaptic input from nonsegmental interneurons. The VI neurons also have commissures; however, their output regions lie in the ventral cord where they synapse onto the ventral musculature; VI dendrites are in the dorsal cord where they receive anatomically defined synaptic input from all three types of DE neurons (J. E. Donmoyer, P. A. Desnoyers, and A. O. W. Stretton, unpublished observations).

Acetylcholine is almost certainly the neurotransmitter released by the DE motoneurons (C. D. Johnson and A. O. W. Stretton, submitted for publication). If there are excitatory cholinergic postsynaptic receptors on the VI dendrite, then the dorsal excitors would excite the VI at the same time that they activate the dorsal muscle. This coupling of inhibition in ventral muscle to excitation in dorsal muscle would provide a mechanism for reciprocity between the dorsal and ventral musculature consistent with the dorsoventral flexions observed during nematode locomotory behavior.

In this paper we use preparations containing two intact commissures, one a dorsal excitor and the other a ventral inhibitor, to investigate interactions between this pair of motoneurons. The results show that the DE-to-VI synapses are indeed excitatory. We also examine the pattern of connectivity 
between each type of dorsal excitor and the inhibitory dendrites and show that each DE neuron excites several VI dendrites. These results provide a physiological map of the region of reciprocal inhibition in the ventral musculature controlled by each type of DE motoneuron. A preliminary report of these findings has appeared (Walrond et al., 1978).

\section{Materials and Methods}

The source and methods of handling adult Ascaris lumbricoides and the basic recording techniques employed in this paper are similar to those described in Walrond et al. (1985).

Dissections. Individual commissures were located and identified in intact animals as described in the previous paper (Walrond et al., 1985). All commissures except those under study were cut. Initially the intact commissures were identified at the end of the physiological experiment by histological analysis of serial $10-\mu \mathrm{m}$ sections (cf. Walrond et al., 1985). In the experiments reported here, however, we often used the responses recorded in muscle cells to establish the identity of the intact neurons (Walrond et al., 1985).
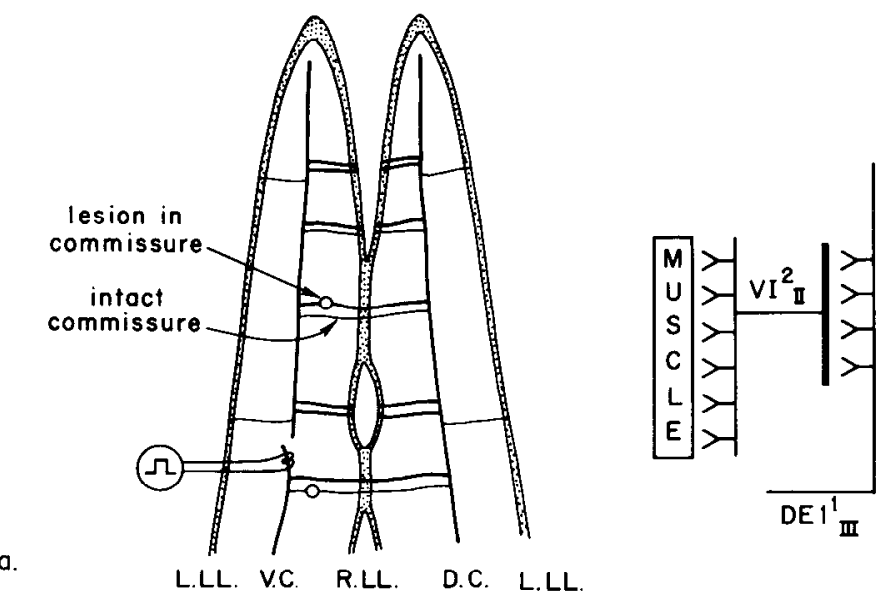

a.

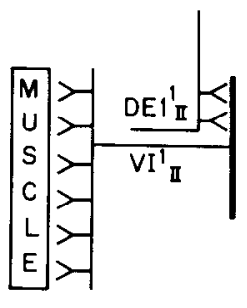

b.

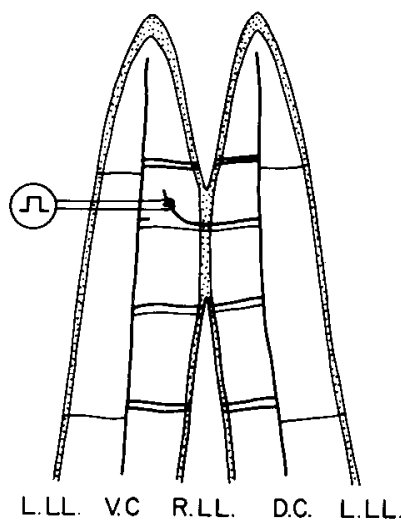

Figure 1. a, Preparation used to study the interaction between DE1 and VI motoneurons. The dorsal and ventral muscle halves remained connected by a DE1 neuron and the next anterior VI neuron. The animal was cut open along the left lateral line ( $L . L L$.), and the right lateral line (R.LL.) was cut so as to leave only two bridges of body wall, each containing a pair of commissures. One member of each pair was severed with an insect pin as indicated by the open circle. A flap of body wall containing a portion of the DE1 neuron in the ventral nerve cord was cut and taken up into a stimulating suction electrode (circle containing a square wave). The right side of the figure diagrammatically shows the relationship between the DE1 neuron and the VI neuron. The Y's represent synaptic output. $b$, Preparation for studying the interaction between $\mathrm{DE} 1$ and VI neurons of the same commissure pair. In this case, the initial segment of DE1 commissure was used for stimulation. V.C., ventral cord; D.C., dorsal cord.

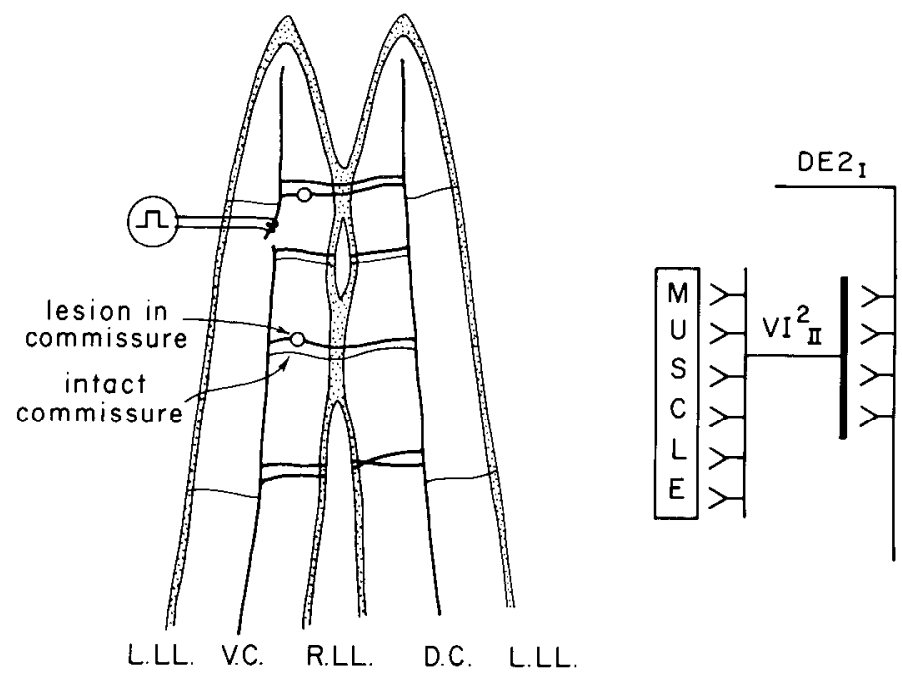

Figure 2. Preparation used to study the interaction between DE2 and VI motoneurons. In this dissection, a DE2 and the second VI commissure of the next posterior segment are intact. Abbreviations and symbols are as in Figure 1.

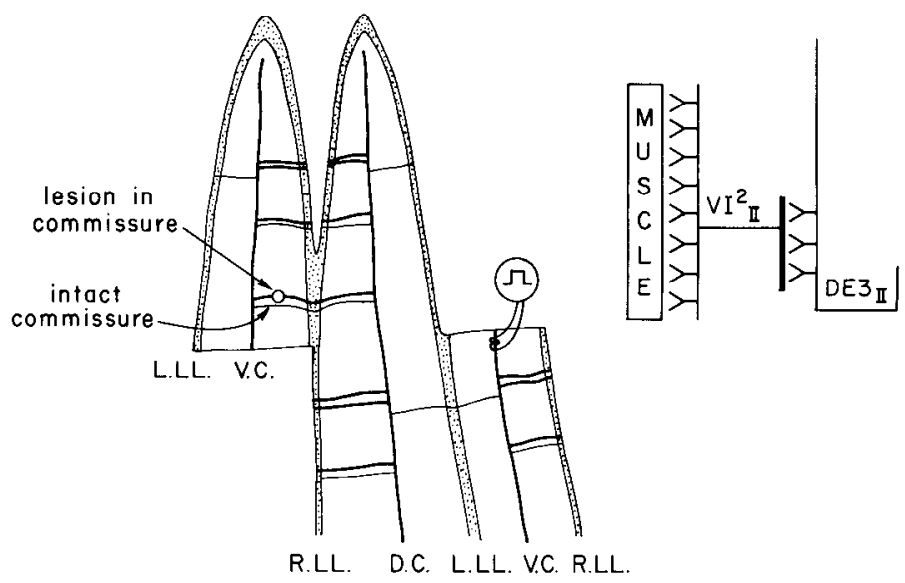

Figure 3. Dissection designed to investigate synaptic interactions between the DE3 and VI motoneurons. In this dissection, a DE3 motoneuron and the second VI motoneuron of the next most anterior segment are intact. Abbreviations and symbols are as in Figure 1.

Interactions between the DE and the VI motoneurons were studied in preparations in which the dorsal and ventral parts of the animal remained connected by one DE motoneuron and one or more VI motoneurons. As in the previous paper (Walrond et al., 1985), the DE neuron was stimulated ventrally using a suction electrode. In order to isolate the stimulation site from the neuromuscular output region of the intact ventral inhibitor(s), the ventral cord was cut so that there was no direct neuronal pathway in the ventral cord between stimulating and recording electrodes (Figs. 1 to 3 ). The only intact neuronal pathway between the stimulation and recording sites was via the dorsal cord.

Due to their different morphologies, investigating the synaptic in teractions between the different types of DEs and the VI neurons required different dissections. The DE1 motoneuron has anterior and posterior processes in the ventral cord and a process in the dorsal cord that projects anteriorly from the commissure. Cutting a flap of ventral body wall containing the anterior limb of a DE1 dendrite permitted stimulation of the DE1 without directly affecting the ventral musculature innervated by more anterior VI neurons as shown in Figure $1 a$. In order to study interactions between DE1 and VI neurons that are members of the same commissure pair, a different dissection was necessary (Fig. 1b). Using sharpened tungsten needles, an incision was made along the body wall between the commissures. A second incision, parallel to the first, yielded a strip of body wall containing part of the commissure of the DE1 neuron. Transecting the strip near the ventral 

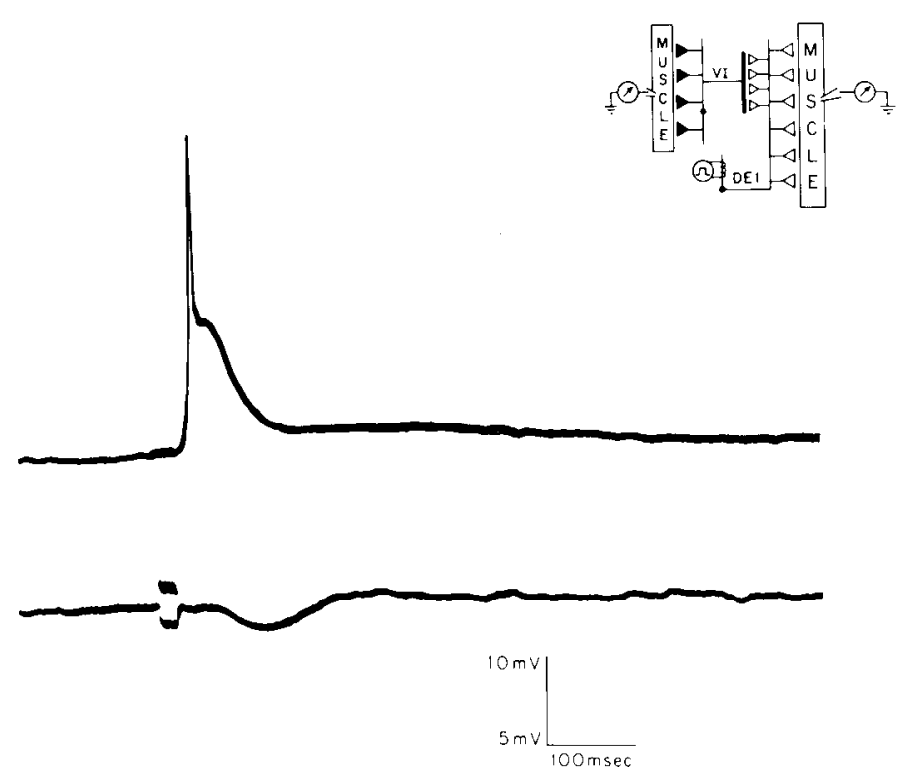

Figure 4. Dorsal and ventral responses evoked by a DE1 motoneuron. Activation of the DE1 motoneuron while simultaneously recording intracellularly in the dorsal and ventral musculature produced a dorsal depolarization (top trace) followed by a ventral hyperpolarization (bottom trace). The inset shows a diagram of the preparation. The circle containing a square wave shows the position of the stimulating electrode. The circles containing arrows represent the positions of intracellular recording electrodes in muscle. Open triangles depict excitatory synaptic output, and solid triangles represent inhibitory synaptic output. Vertical scale: $10 \mathrm{mV}$, upper trace, $5 \mathrm{mV}$, lower trace.
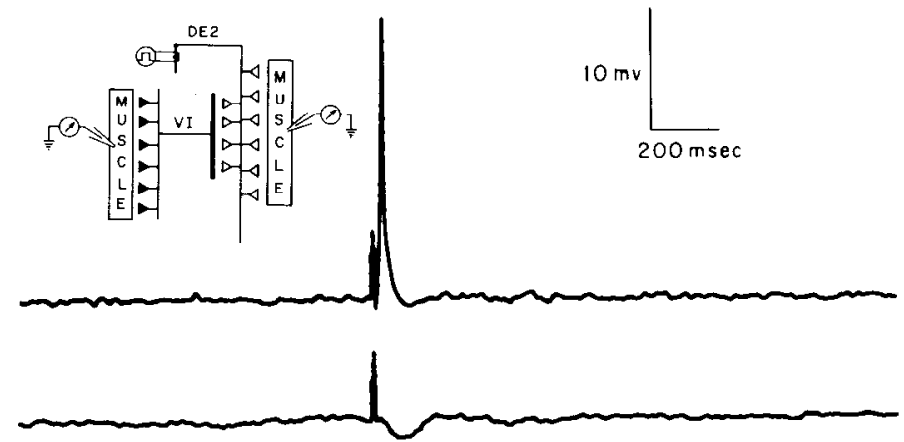

Figure 5. Interaction between DE2 and VI motoneurons. Activation of the DE2 neuron produced a depolarization (spike) in the dorsal musculature (top trace) followed by a hyperpolarization in the ventral musculature (bottom trace). The inset shows a diagram of the preparation. For details concerning the symbols, see Figure 4 legend.

nerve cord produced a flap which could be taken up into a suction electrode for stimulation.

The preparation used for investigating the DE2 neurons is shown in Figure 2. Since the dendrites and axons of the DE2 neuron lie posterior to their commissures, a posterior flap containing the DE2 dendrite was dissected, and we studied the evoked response in ventral muscle cells posterior to the cut in the ventral cord.

A preparation in which the dorsal and ventral muscle halves remained connected by one DE3 neuron and one or more VI neurons is shown in Figure 3. The DE3 neuron has a left-hand commissure, whereas VI neurons have right-hand commissures. The animal was cut along the right lateral line to a level just anterior to where the DE3 neuron enters the dorsal cord. A cut from the left to the right lateral line was then made through the ventral half of the animal and then extended anteriorly along the left lateral line. A flap of tissue containing the nerve cord was cut anterior to where the DE3 neuron leaves the ventral cord and was taken up into a suction electrode for stimulation.

Electrophysiological techniques. Intracellular recordings from dorsal and ventral muscle cells which lay in the output zones of the intact DE and VI motoneurons, respectively, were used for simultaneous moni-
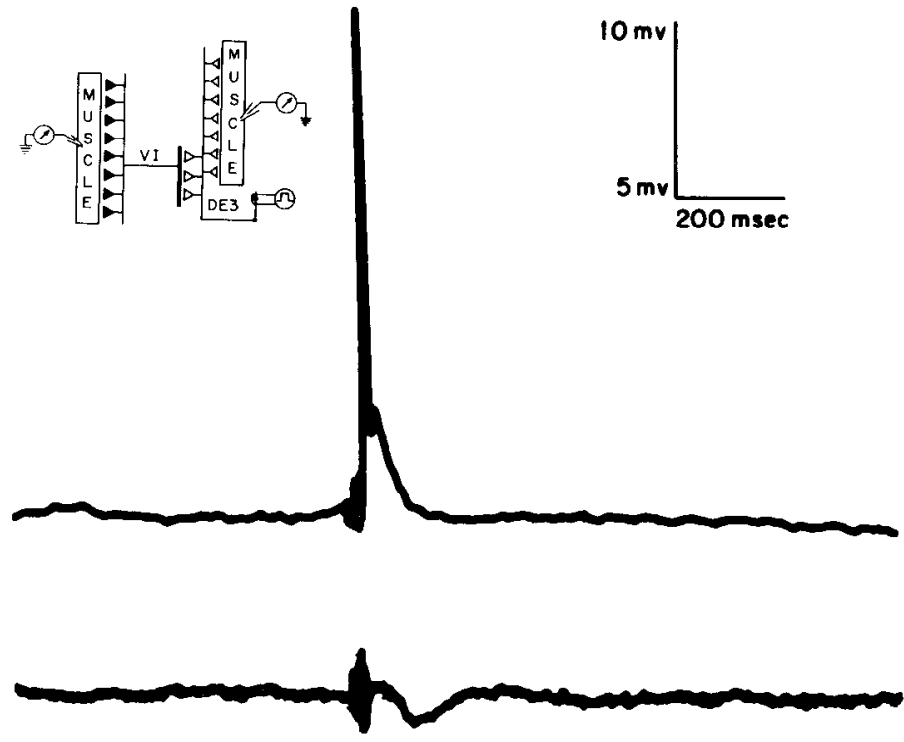

Figure 6. Dorsal and ventral responses evoked by the DE3 neuron. Ventrally activating the DE3 neuron produced a dorsal depolarizing response followed by a ventral hyperpolarizing response. Vertical scale: $10 \mathrm{mV}$, upper trace; $5 \mathrm{mV}$, lower trace. The inset shows a diagram of the preparation. For symbols, see the legend to Figure 4.

TABLE I

Synaptic interactions between dorsal excitatory and ventral inhibitory motoneurons

The first column lists the DE-VI combination investigated. The second column shows the number of experiments in which activation of the VI motoneuron was observed, and the numbers in parentheses show the number of experiments carried out for each combination. The third and fourth columns give the number of experiments in which the VI or DE neuron was cut while recording evoked responses.

\begin{tabular}{|c|c|c|c|}
\hline \multirow{2}{*}{$\begin{array}{l}\text { DE-VI Interaction } \\
\text { Investigated }\end{array}$} & \multirow{2}{*}{$\begin{array}{l}\text { No. of Experiments } \\
\text { in which VI Activa- } \\
\text { tion was Observed }\end{array}$} & \multicolumn{2}{|c|}{ No. of Cuts } \\
\hline & & VI & $\mathrm{DE}$ \\
\hline $\mathrm{DE} 1_{1 \mathrm{I}}^{1} \rightarrow \mathrm{VI}_{\mathrm{I}}^{2}$ & $10(10)$ & 1 & 1 \\
\hline $\mathrm{DE} 1_{\mathrm{II}}^{1} \rightarrow \mathrm{VI}_{\mathrm{II}}^{1}$ & $4(4)$ & & \\
\hline $\mathrm{DE} 1_{\mathrm{II}}^{2} \rightarrow \mathrm{VI}_{\mathrm{II}}^{1}$ & $13(13)$ & 4 & 1 \\
\hline $\mathrm{DE} 1_{\mathrm{II}}^{2} \rightarrow \mathrm{VI}_{\mathrm{II}}^{2}$ & $6(6)$ & $2^{a}$ & 2 \\
\hline $\mathrm{DE} 1_{\text {III }}^{1} \rightarrow \mathrm{VI}_{\text {II }}^{2}$ & $8(8)$ & 2 & 2 \\
\hline $\mathrm{DE} 1_{\mathrm{III}}^{1} \rightarrow \mathrm{VI}_{\mathrm{III}}^{2}$ & $3(3)$ & & \\
\hline $\mathrm{DE} 1_{\mathrm{III}}^{2} \rightarrow \mathrm{VI}_{\mathrm{III}}^{1}$ & $4(4)$ & & 1 \\
\hline $\mathrm{DE} 2_{\mathrm{I}} \rightarrow \mathrm{VI}_{\mathrm{II}}^{1}$ & $18(18)$ & 5 & \\
\hline $\mathrm{DE} 2_{\mathrm{I}} \rightarrow \mathrm{VI}_{\mathrm{II}}^{2}$ & $14(14)$ & 6 & 2 \\
\hline $\mathrm{DE} 2_{1} \rightarrow \mathrm{VI}_{1 \mathrm{II}}^{1}$ & $2(5)$ & & 1 \\
\hline $\mathrm{DE} 2_{\mathrm{II}} \rightarrow \mathrm{VI}_{\mathrm{III}}^{1}$ & $7(7)$ & 2 & 2 \\
\hline $\mathrm{DE} 2_{\mathrm{II}} \rightarrow \mathrm{VI}_{\mathrm{III}}^{2}$ & $4(4)$ & 2 & 1 \\
\hline $\mathrm{DE} 3_{1} \rightarrow \mathrm{VI}_{\mathrm{I}}^{2}$ & $5(5)$ & 3 & \\
\hline $\mathrm{DE} 3_{\mathrm{II}} \rightarrow \mathrm{VI}_{\mathrm{II}}^{1}$ & $8(8)$ & 5 & \\
\hline $\mathrm{DE}_{\mathrm{II}} \rightarrow \mathrm{VI}_{\mathrm{II}}^{2}$ & $1(5)$ & 1 & \\
\hline
\end{tabular}

${ }^{a}$ Both members of the pair were cut.

toring of the output of both intact motoneurons. Either square wave single pulses or trains of pulses were used to stimulate the dendrite of the excitatory motoneuron. In cases where large amplitude VI responses were not observed, signal averaging was routinely used to detect possible low amplitude signals. Some experiments employed bipolar wirestimulating electrodes positioned over the dendrite of the excitatory motoneuron. These experiments yielded results similar to experiments in which suction electrodes were used as described above. 

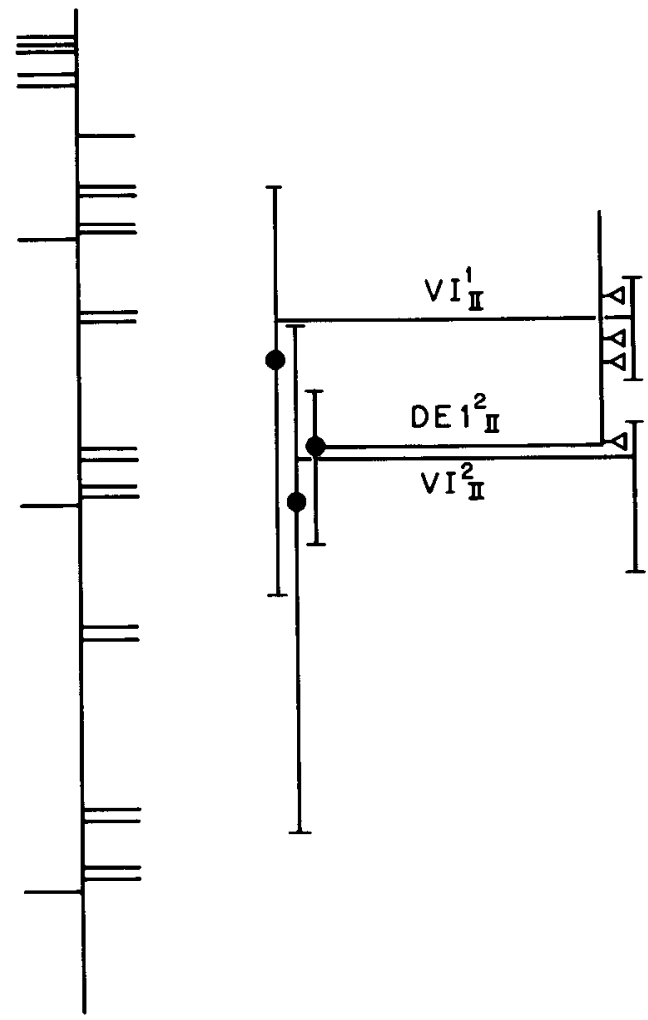

Figure 7. A diagrammatic representation of synaptic interactions between a DE1 neuron and more anterior VI neurons. The DE1 neurons are present in two copies per segment. Each DE1 neuron synapses onto two VI neurons, the one with which it enters the dorsal cord, and the next anterior one. The diagram on the left shows the array of commissures in the first three segments drawn in register with the diagram on the right.

Nomenclature. Each segment contains two DE1, VI, V-1, and V-2 neurons. In order to distinguish them, they are given superscripts (e.g., $\mathrm{DE} 1^{1}$ and $\mathrm{DE} 1^{2}$, denoting the anterior and posterior neurons in each segment, respectively). The segments of origin are given as Roman numeral subscripts; for example, DE1 $1_{\mathrm{II}}^{2}$ is the posterior DE1 neuron of the second segment.

\section{Results}

In preparations where the dorsal and ventral nerve cords remained connected by the commissures of two motoneurons, one a dorsal excitor and the other a ventral inhibitor, stimulation of the excitor evoked a depolarizing potential in the dorsal musculature followed, after a delay, by a hyperpolarizing potential in the ventral musculature. Activation of DE1, DE2, or DE3 neurons evoked similar responses (Figs. 4 to 6 ). Since the DF-to-VI synapse provided the only intact neuronal pathway between the ventral site of activation of the $\mathrm{DE}$ neuron and the intracellular recording site in the ventral musculature, these experiments demonstrate that DE motoneurons make excitatory synapses onto VI motoneurons.

Synaptically activated ventral hyperpolarizations were similar to hyperpolarizations evoked by the direct electrical activation of the VI dorsal dendrite (Walrond et al., 1985). Trains of stimulating pulses generally elicited larger ventral hyperpolarizing responses than did single pulses.

To ensure that both dorsal and ventral evoked responses do indeed depend on the DE-to-VI synapse, rather than being produced by current spread from the stimulating electrode, in many experiments we cut one of the two intact commissures while recording the evoked responses (Table I). Cutting the commissure of the dorsal excitor eliminated both the dorsal depolarizing response and the ventral hyperpolarizing response.
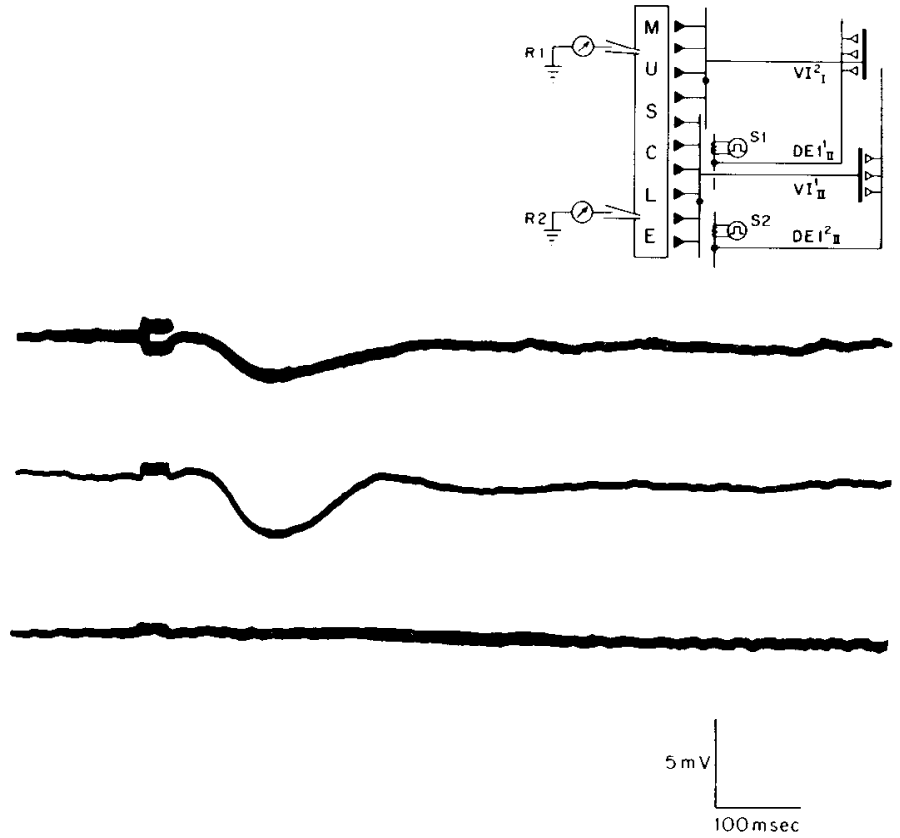

Figure 8. The $\mathrm{DE} 1 \mathrm{II}$ neuron activates the $\mathrm{VI}_{\mathrm{II}}^{1}$ neuron but not the $\mathrm{VI}_{1}^{2}$ neuron. $R 1$ and $R 2, S 1$ and $S 2$ are recording and stimulating electrodes, respectively. Stimulation of DE1 1 II synaptically activated $\mathrm{VI}_{\mathrm{II}}^{1}$ (top trace recording from $\mathrm{R} 2$ and stimulating at $\mathrm{S} 2$ ), and stimulation of $\mathrm{DE}_{1}^{1}{ }_{\text {II }}$ activated $\mathrm{VI}_{\mathrm{I}}^{2}$ (middle trace recording from $\mathrm{R} 1$ and stimulating at $\mathrm{S} 1$ ). However, stimulation of $\mathrm{DE} 1_{\mathrm{II}}^{2}$ failed to activate $\mathrm{VI}_{1}^{2}$ (bottom trace recording from $\mathrm{R} 1$ and stimulating at $\mathrm{S} 2$ ).

Severing the commissure of the ventral inhibitor eliminated the ventral hyperpolarization but left the dorsal depolarization intact. Thus, an intact excitatory pathway to the dorsal cord is necessary to evoke the ventral inhibition.

Each DE motoneuron is capable of activating several VI motoneurons. The specificity of these connections was investigated in preparations where various combinations of DE and VI neurons remained intact (Table I). From the results of these experiments we constructed neuronal maps (Figs. 7, 9, and 11) which show the synaptic interactions between the DE and VI motoneurons. Each DE1 neuron excites two VI neurons, the one with which it enters the dorsal cord and the next anterior VI neuron (Fig. 7); the second DE1 neuron in a segment drives both the $\mathrm{VI}^{1}$ and the $\mathrm{VI}^{2}$ neurons of the same segment, whereas the first DE1 neuron in a segment drives the $\mathrm{VI}^{1}$ neuron of the same segment as well as the $\mathrm{VI}^{2}$ neuron located in the next anterior segment.

The DE1 motoneuron enters the dorsal cord and projects anteriorly, overlapping the dendrites of three, and sometimes four, VI dendrites. Anatomical studies have shown that this dorsal process can be divided into two distinct regions. The region proximal to the commissure makes numerous neuromuscular synapses and overlaps the processes of two VI dendrites. Anterior to this there is a region where neuromuscular synapses are rare (A. O. W. Stretton, R. M. Fishpool, E. Southgate, and J. E. Donmoyer, unpublished observation). This anterior portion overlaps a third, and sometimes a fourth, VI dendrite. In order to test whether DE1 neurons make functional connections that activate these more anterior VI neurons, experiments were performed in preparations where four commissures remained intact (Fig. 8). As expected, the DE $1_{11}^{1}$ neuron activated the $\mathrm{VI}_{\mathrm{I}}^{2}$ neuron, and the $\mathrm{DE} 1_{\mathrm{II}}^{2}$ neuron activated the $\mathrm{VI}_{\mathrm{II}}^{1}$ neuron. However, when stimulating the $\mathrm{DE} 1_{\mathrm{II}}^{2}$ neuron, we failed to observe activity in the $\mathrm{VI}_{I}^{2}$ neuron even when signal averaging was used to detect weak signals. We 


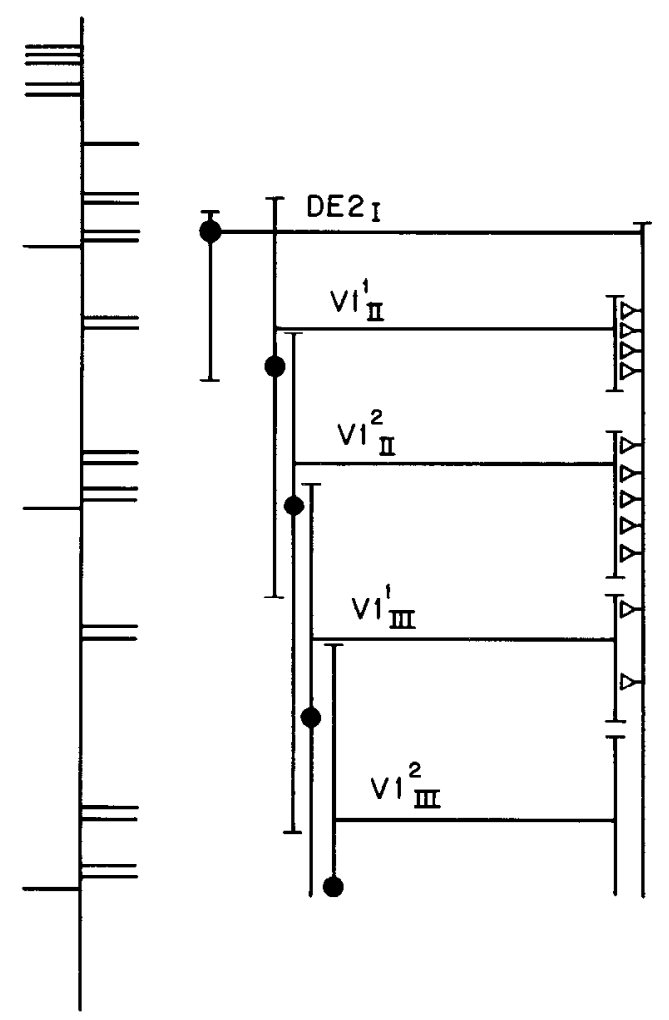

Figure 9. Interaction between a DE2 neuron and more posterior VI neurons. The $\mathrm{DE} 2_{\mathrm{I}}$ neuron synapses onto the next three posterior VI neurons. A weak response of the $\mathrm{VI}_{\mathrm{III}}^{1}$ neuron was revealed by signal averaging techniques in two of five experiments. No interaction with the $\mathrm{VI}_{\text {III }}^{2}$ was observed. The diagram on the left shows the array of commissures in the first three segments drawn in register with the diagram on the right.

therefore conclude that the DE1 neurons activate only those VI dendrites lying within their neuromuscular output region.

Stimulating the DE2 neuron in preparations containing various intact VI neurons produced a map of synaptic interactions between these two neuron types (Fig. 9). Stimulating the DE2 neurons of the first or second segments produced ventral hyperpolarizations when either of the next two posterior VI neurons ( $\mathrm{VI}^{1}$ and $\mathrm{VI}^{2}$ of the next posterior segment) remained intact. In two of five experiments, DE2 1 activated a third VI neuron ( $\mathrm{VI}_{\mathrm{III}}^{1}$ ); although this response was weak, it was clearly revealed using signal averaging. No activation of the VIIII was observed.

The axon of the DE2 neuron overlaps with the posterior limb of the next more anteriorly located $\mathrm{VI}^{2}$ neuron. Experiments designed to investigate the possible interactions between these two neurons employed preparations containing four intact commissural neurons (Fig. 10). The ventral activation of the DE2 neuron failed to produce ventral hyperpolarizations through the more anterior VI neuron.

Experiments employing combinations of DE3 and VI neurons revealed that the ventral activation of a DE3 neuron evoked ventral hyperpolarizing potentials through the $\mathrm{VI}^{2}$ neuron of the same segment (Fig. 11). Interactions between the DE3 neuron and the first VI neuron of the same segment were observed only once in five experiments. Since synapses from DE3 onto $\mathrm{VI}^{1}$ have been observed by electron microscopy, the reasons why the DE3 neuron failed to drive this VI neuron consistently remain obscure. In general, the DE3 neurons are more difficult to activate than are the other excitors, and activation of the DE3 neuron frequently failed to evoke re- sponses from muscle cells in the more anterior portion of its neuromuscular output region.

\section{Discussion}

In the experiments described in this paper, activity in excitatory and inhibitory motoneurons of the nematode Ascaris lumbricoides has been monitored by recording from the muscle cells they innervate. We show that stimulating single $\mathrm{DE}$ motoneurons evokes not only depolarizing responses in dorsal muscle cells but also hyperpolarizing responses in ventral muscle cells. As we reported in the preceding paper (Walrond et al., 1985), the depolarizing responses are excitatory, producing action potentials in muscle, whereas the hyperpolarizing responses are inhibitory, suppressing spontaneous muscle action potentials.

The ventral response requires that the commissures of two motoneurons, one a DE and the other a VI, be intact. Since ultrastructural studies have demonstrated a monosynaptic connection from DE to VI motoneurons in the dorsal nerve cord (P. A. Desnoyers, J. E. Donmoyer, and A. O. W. Stretton, unpublished observation), this monosynaptic pathway is almost certainly responsible for the physiological responses described in this paper. These DE-to-VI motoneuron pathways provide a mechanism for reciprocal inhibition whereby neuronal activation of the dorsal musculature provides concomitant inhibition of the ventral musculature.

By physiological criteria, each VI neuron receives synaptic input from several DE motoneurons. The first VI neuron in each segment is activated by two DE1 neurons, one or two DE2 neurons (the input from the more anteriorly situated DE2 neuron is variable and weak), and zero or one DE3 neuron. The second VI neuron in each segment is activated by two DE1 neurons, one DE2 motoneuron, and one DE3 neuron (Fig. 12). All of these synaptic connections have been observed by electron microscopy, with the exception of the contacts between the DE1 and VI neurons which enter the dorsal nerve cord together (i.e., the $\mathrm{DE} 1^{1}$-to- $\mathrm{VI}^{1}$ and the $\mathrm{DE} 1^{2}$-to- $\mathrm{VI}^{2}$ contacts).

Synapses between $\mathrm{DE} 3$ and $\mathrm{VI}^{1}$ neurons have also been observed by electron microscopy, yet we found physiological activity due to this synapse in only one of five experiments. This variability might reflect differences in synaptic strength or frequency in different animals. Since the DFs neurons frequently fail to activate muscles in the anterior part of their neuromuscular output zones (Walrond and Stretton, 1985), we believe that a more likely explanation is that activity in the DE3 axon often fails to propagate into its more anterior regions. Whether these effects are intrinsic to the DE3 neuron or simply reflect our inability to activate it properly remains to be elucidated.

The mapping experiments also eliminate the formal possibility that VI dendrites can be excited by active muscle cells. Even though evoked excitatory activity in muscle cells may spread outside of the output region of a stimulated motoneuron through the electrical synapses between muscle cells (Walrond and Stretton, 1985), each DE neuron excites only those VI dendrites that lie within its output region (i.e., the region where it makes neuromuscular synapses).

Ultrastructural studies have revealed rare synapses between two DE neurons. However, experiments described in this paper and others presented in the following paper (Walrond and Stretton, 1985) indicate that DE motoneurons fail to interact strongly. If a DE neuron were capable of driving other DE neurons, activating any one of the DE cells would have produced a more extensive inhibition of the ventral musculature than we observed. White et al. (1976) have described electrical synapses between homologous (e.g., DE2 $2_{1}$ and $\mathrm{DE} 2_{1 \mathrm{II}}$ motoneurons in Caenorhabditis elegans. At present, there is no evidence for contacts of this kind from ultrastructural studies in Ascaris 


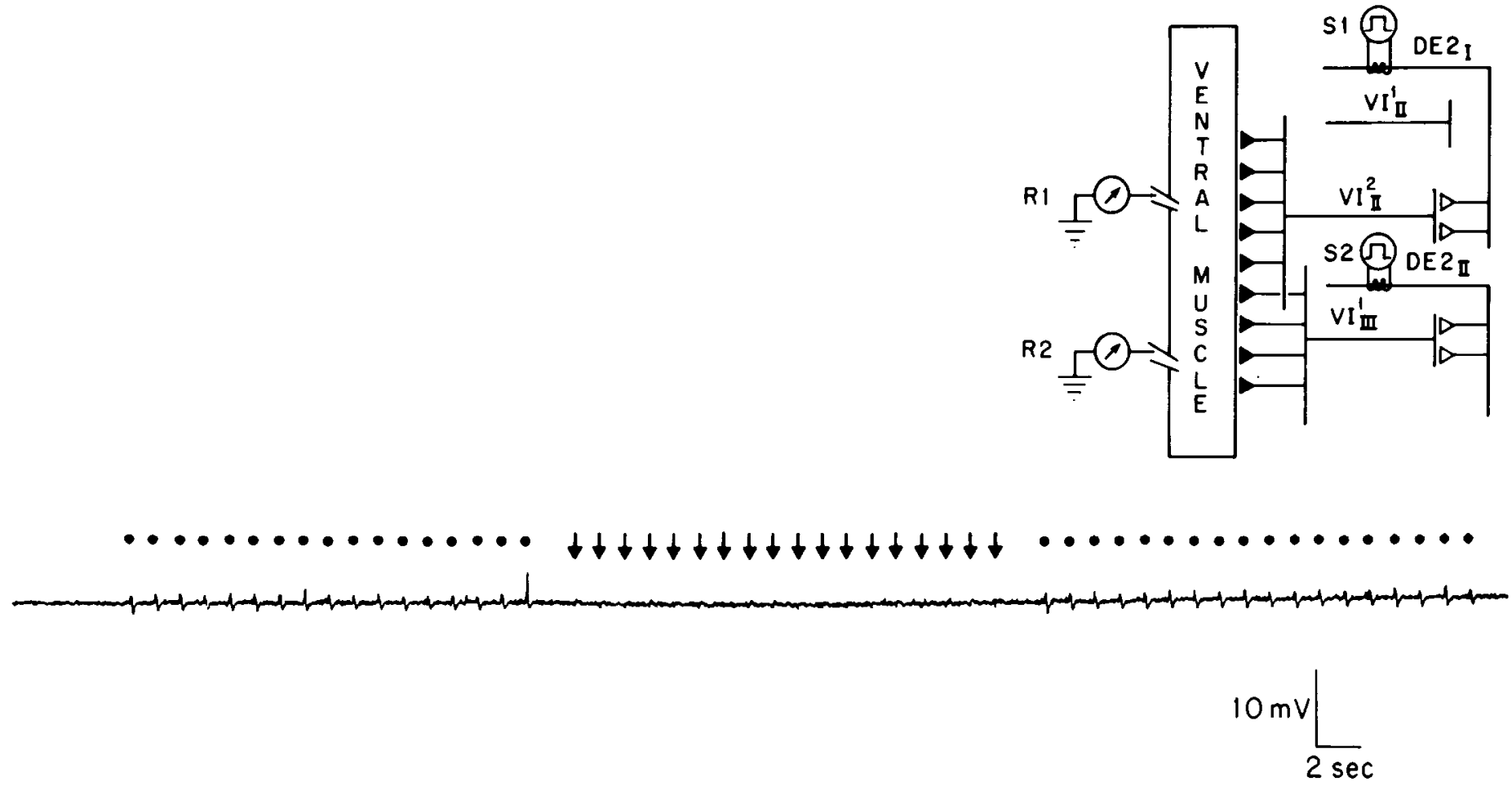

Figure 10. DE2 neurons interact with only more posterior VI neurons. Recordings taken at $R 1$ show that stimulating the DE2 ${ }_{\mathrm{I}}$ neuron activates the $\mathrm{VI}_{\mathrm{II}}^{2}$ neuron (each dot represents a stimulus applied through $S 1$ ), but that stimulating the DE2 $2_{\mathrm{II}}$ neuron fails to activate the VIII neuron (each arrow represents a stimulus applied through $S 2$ ). Recordings (not shown) taken from $R 2$ demonstrated that stimulating the DE2 $2_{\mathrm{II}}$ neuron activated the $\mathrm{VI}_{\mathrm{III}}^{\mathrm{a}}$ neuron.

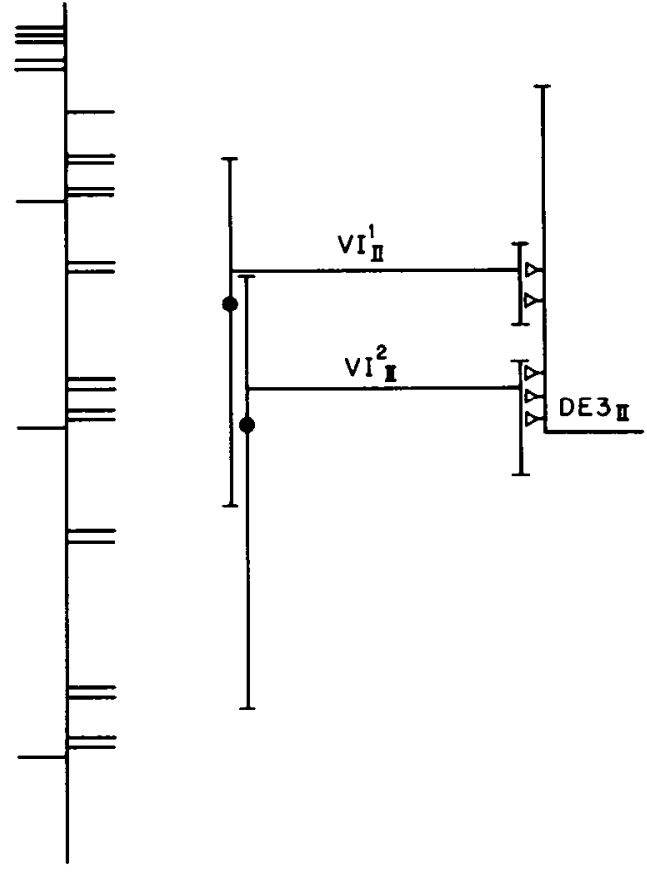

Figure 11. A diagrammatic representation of synaptic interactions between the DE3 neuron and more anterior VI neurons. The process of the DE3 $3_{\mathrm{II}}$ neuron extends anteriorly in the dorsal cord. It strongly activated the next anterior VI neuron ( $\mathrm{VI}_{\mathrm{II}}^{2}$ ) but showed variable activation of the $\mathrm{V} \mathrm{I}_{\mathrm{II}}^{\mathrm{I}}$ neuron.

(J. E. Donmoyer, P. A. Desnoyers, and A. O. W. Stretton, unpublished observation). Our physiological experiments show that, if such contacts do exist in Ascaris, they are not strong enough to produce a detectable response in the muscle cells or the VI neurons innervated by the postsynaptic DE motoneurons.

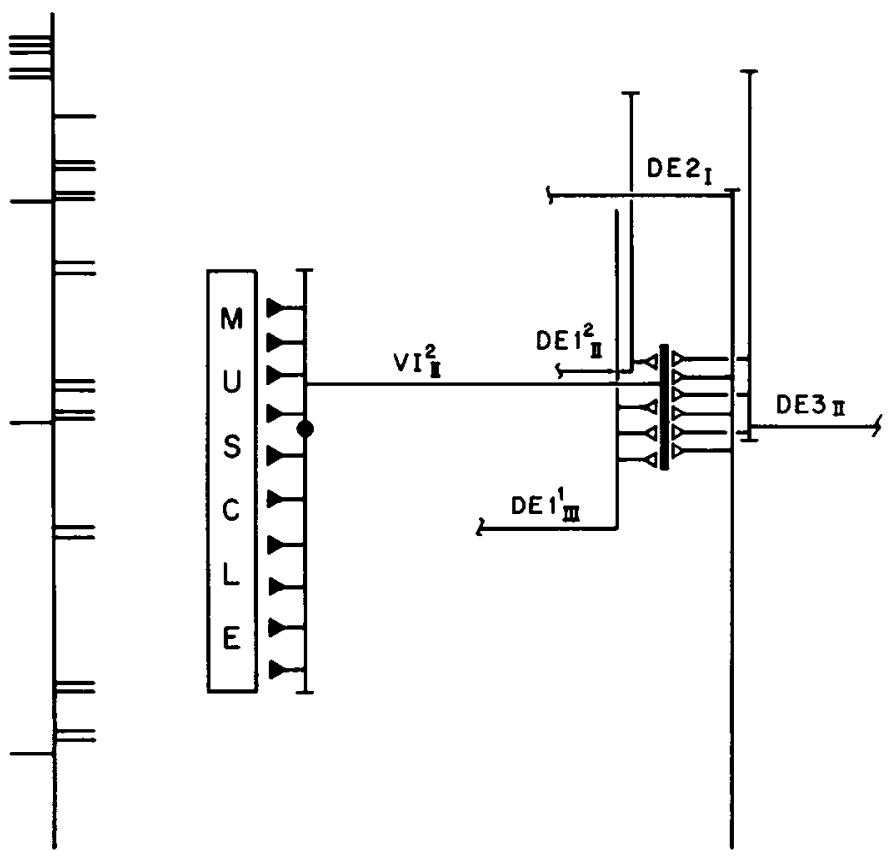

Figure 12. Synaptic input onto the dorsal dendrite of a VI neuron. The $\mathrm{VI}_{11}^{2}$ neuron receives synaptic input from two DE1 neurons, one DE2 neuron, and one DE3 neuron.

Nematode locomotory behavior consists of propagated waves of contraction in the ventrodorsal plane (Crofton, 1966). The synaptic interactions described in this paper provide a system that mediates reciprocity between dorsal and ventral muscles, so that a neuronally evoked dorsal contraction is complemented by neuronally evoked ventral inhibition. The region of a dorsally activated muscle is opposite the ventrally inhibited region. Under this system, the animal can throw a specific region of 
its body into a dorsal bend. Electron microscopic results show that a corresponding circut exists in the ventral nerve cord $(J$. E. Donmoyer, P. A. Desnoyers, and A. O. W. Stretton, unpublished observation). The V-1 and V-2 neurons innervate the ventral musculature and also synapse onto the dendrites of the DI motoneurons. These ventral synaptic interactions have not been investigated physiologically, but if, as we expect, the V-1 and V-2 motoneurons are excitatory (cf "Discussion" in Walrond et al., 1985), and if their synapses with DI are physiologically similar to those of DE motoneurons with VI dendrites in the dorsal nerve cord, these interactions would provide a system whereby the animal produces a ventral bend, i.e., a ventral contraction complemented by a region of dorsal inhibition.

A nematode moves by smoothly propagating a waveform consisting of alternating dorsoventral and ventrodorsal contractions along its body. At a particular point in the body, as the waveform progresses, the state of the musculature must change from maximal dorsal excitation to maximal ventral excitation in a cyclic fashion. There are no obvious discontinuities in this cycle. The reciprocal inhibitory circuits provide a series of discrete sites where a region of dorsal contraction may be complemented by a region of ventral inhibition and vice versa. If a series of such circuits were activated sequentially, a wave form could be propagated along the body. The mechanisms by which propagating waves move without any discontinuity is not clear. However, we know that in Ascaris, the output zones of adjacent members of the same class of $\mathrm{DE}$ or DI motoneurons overlap (Stretton et al., 1978), so that waxing and waning of activity in neighboring circuits might lead to smooth transitions as the waveform progressed. It is also possible that the mechanical properties of the muscle, and/or hypodermis and cuticle, could act as a smoothing mechanism. Alternatively, some dynamic interaction between the neurons might vary the state of excitation and inhibition in a continuous way; indeed, anatomical experiments have shown that reciprocal synapses occur between the processes of DE and DI neurons where they make neuromuscular synapses (P. A. Desnoyers, J. E. Donmoyer, and A. O. W. Stretton, unpublished observation), so that graded local circuit interactions are highly probable.

\section{References}

Bullock, 'T. M., and G. A. Horridge (1965) Structure and Function in the Nervous System of Invertebrates, W. H. Freeman and Co., San Francisco.

Crofton, H. D. (1966) Nematodes, Hutchinson University Library, London.

Hesse, R. 1892. Uber das Nervensystem von Ascaris megalacephala. Z. Wiss. Zool. 54: 548-568.

Stretton, A. O. W., R. M. Fishpool, E. Southgate, J. E. Donmoyer, J. P. Walrond, J. E. R. Moses, and I. S. Kass (1978) Structure and physiological activity of the motorneurons of the nematode Ascaris. Proc. Natl. Acad. Sci. U. S. A. 75; 3493-3497.

Walrond, J. P., and A. O. W. Stretton (1985) Excitatory and inhibitory activity in the dorsal musculature of the nematode Ascaris evoked by single dorsal excitatory motoneurons. J. Neurosci. $5: 16-22$.

Walrond, J. P., J. E. Donmoyer, P. A. Desnoyers, and A. O. W. Stretton (1978) Synaptic interactions between motorneurons of Ascaris. Soc. Neurosci. Abstr. 4: 209.

Walrond, J. P., I. S. Kass, A. O. W. Stretton, and J. E. Donmoyer (1985) Identification of excitatory and inhibitory motoneurons in the nematode Ascaris by electrophysiological techniques. J. Neurosci. 5 . $1-8$.

White, J. G., E. Southgate, J. N. Thomson, and S. Brenner (1976) The structure of the ventral nerve cord of Caenorhabditis elegans. Philos. Trans. R. Soc. Iond. (Biol.) 275: 327-348. 